\title{
Recursos digitales para la traducción jurídica en el campo medioambiental (italiano-español)
}

\author{
Rubén González Vallejo ${ }^{1}$
}

Recibido: 7 de septiembre de 2020 / Modificado: 25 de enero de 2021 / Aceptado: 3 de marzo de 2021

Resumen. El presente trabajo se centra en la importancia de la documentación en la traducción del medio ambiente al suponer una novedad en el campo formativo y un reto por sus características específicas. Inicialmente, se delinearán las competencias de la traducción especializada para resaltar con posterioridad la intertextualidad e interdisciplinariedad del sector medioambiental que el traductor debiera conocer para una búsqueda terminológica más eficaz. Posteriormente, se resaltará la situación legislativa medioambiental en España e Italia como paso previo a la presentación de los recursos lingüísticos y terminológicos disponibles en Internet. Estos últimos abarcarán desde fuentes normativas hasta complementarias en la combinación italiano-español y supondrán un válido enfoque para la satisfacción del documento final, pues la competencia documental se postula como uno de los pilares fundamentales del ejercicio de la profesión.

Palabras clave: medio ambiente; recursos digitales; recursos documentales; traducción jurídica.

\section{[en] Digital resources for legal translation in the environmental field (Italian- Spanish)}

\begin{abstract}
This paper focuses on the importance of the literature review in the translation of environmental texts, since it is a novelty in the field of training and its specific characteristics make it challenging. Firstly, the competencies of specialised translation will be outlined in order to subsequently highlight the intertextuality and interdisciplinary nature of the environmental sector, which the translator should be aware of in order to carry out a more efficient terminological search. Afterwards, the environmental legislative situation in Spain and Italy will be highlighted as a preliminary step before the presentation of the linguistic and terminological resources available on the Internet. The latter will range from normative to complementary sources in the Italian-Spanish combination and will represent a valid approach to the completion of the final document, since documentary competence stands as one of the fundamental pillars for the exercise of the profession.
\end{abstract}

Key words: environment; digital resources; documentary resources; legal translation.

Índice: 1. Introducción: cambios que ha sufrido el traductor 2. La documentación en traducción especializada 3. La traducción del medio ambiente 4. Recursos digitales para la traducción jurídica 4.1. Bases de datos jurídicas 4.2. Documentos normativos 4.3. Material divulgativo temático de principal interés 4.3.1. Revistas jurídicas sobre medio ambiente 4.3.2. Revistas divulgativas sobre medio ambiente 4.4. Instituciones y asociaciones de medio ambiente 4.5. Portales y páginas web medioambientales 4.6. Recursos terminológicos 4.6.1. Glosarios monolingües y multilingües 4.6.2. Bases terminológicas 5. Conclusiones 6. Bibliografía. 
Cómo citar: González Vallejo, R. (2021): «Recursos digitales para la traducción jurídica en el campo medioambiental (italiano-español)», Cuadernos de Filología Italiana, 28, pp. 73-88.

\section{Introducción: cambios que ha sufrido el traductor}

No es baladí la cuestión sobre cómo ha cambiado la figura del traductor con el advenimiento de las nuevas tecnologías y el camino que ha abierto las puertas de la globalización. El uso de herramientas informáticas representa un elemento interdisciplinar cada vez más requerido al traductor, pues de ello dependerá en parte su rapidez y calidad en la traducción de textos que requieran un elevado volumen de trabajo y una cierta homogeneización. A tal respecto, la oferta y demanda de cursos sobre las llamadas herramientas TAO (Traducción Asistida por Ordenador) han suscitado un gran interés y las universidades en las carreras de Traducción prevén en sus planes de estudio asignaturas dedicadas a su uso. En ellas, la búsqueda de recursos documentales recubre una exhaustiva importancia en el resultado final de la traducción, pues la competencia documental se postula como uno de los grandes aliados del traductor. Asimismo, el traductor especializado dispone a su alcance de un amplio abanico de fuentes terminológicas gracias al creciente intercambio de archivos en línea. Por ello, en el presente trabajo abordaremos los recursos documentales que podemos encontrar en la traducción jurídica en el campo medioambiental al representar uno de los temas más preocupantes de nuestro siglo y más dinámico debido a la cantidad temática que abarca.

\section{La documentación en traducción especializada}

La traducción especializada no diverge significativamente de la no sectorial al estar compuesta por diferentes subcompetencias, desde la búsqueda y recogida de información inicial hasta el proceso de traducción en sí, junto a la revisión y edición final del documento (García 2001). Sin embargo, sí subyacen competencias específicas relacionadas con el ámbito sectorial del texto en cuanto al análisis de su macroestructura y microestructura y con la búsqueda de técnicas y estrategias de traducción (piénsese por ejemplo en el análisis de la pormenorizada división interna de una ley de modificaciones o en los elementos técnicos para tener en cuenta en una traducción jurada).

Cuando hablamos de competencia, nos referimos a la «macrocompetencia que constituye el conjunto de capacidades, destrezas, conocimientos e incluso actitudes que reúnen los traductores profesionales y que intervienen en la traducción como actividad experta» (Kelly 2002: 14). Las competencias más relacionadas directamente con la búsqueda de recursos documentales son, para esta autora, la temática y la instrumental profesional. La primera de ellas abarca el conocimiento sobre los temas sectoriales que permiten a la figura del traductor comprender el documento de partida. Ello conllevaría la adquisición de un acervo teórico sobre el elemento interdisciplinar que subyace en el texto: en nuestro caso lo representaría el medio ambiente y su estudio pasaría desde los recursos normativos para analizar cómo se recoge el medio ambiente tanto en la legislación de ambos países (v. §4) hasta las 
fuentes divulgativas que permitan adquirir el vocabulario específico y el funcionamiento de los elementos que lo componen. A tal propósito, los papeles de aprendiz e investigador en el traductor para Mayoral (2005) serían un perfecto reflejo del profesional que busca siempre conocimientos especializados que aprender para ampliar su bagaje lingüístico-cultural. Por su parte, la competencia instrumental comprende el manejo de herramientas informáticas relacionadas con la traducción y las fuentes de documentación que el traductor necesita para llevar a cabo su cometido. En este aspecto, el acabado final que otorgan los instrumentos informáticos a su alcance proporciona al traductor el papel de artesano (Mayoral 2005). Por otra parte, esta competencia también cubre el espectro práctico de la profesión, es decir, conocer los aspectos del código de conducta o la facturación por los servicios prestados. Esta competencia está íntimamente relacionada además con la extralingüística, la cual ayudará al traductor a través de su conocimiento cultural a seleccionar aquellos elementos pertinentes que puedan resolver los problemas de equivalencia con la mayor solvencia posible.

Por último, resaltamos una competencia específica nada desdeñable que denominaremos relacional, vista la flexibilidad de los textos jurídicos para entrelazar textos tanto interna como externamente en su cometido de abarcar todas las casuísticas posibles. La intertextualidad ha sido puesta de manifiesto por varios autores (Marinkovich 1998; Mendoza 1993; Macedo 2008) y representa un escollo en la documentación jurídica, pues la elevada relación entre leyes heterogéneas, muchas veces innecesaria, obstaculiza la labor del traductor (González Vallejo, 2021). Como ejemplo expondremos cuatro niveles de intertextualidad extraídos de la Ley de modificación italiana de 22 de mayo de 2015 n. 68 sobre medio ambiente. Inicialmente encontramos referencias intertextuales explícitas, que se explican por la aparición de citaciones literales de leyes externas junto al nuevo añadido, como en el caso del art 1.4, «All'articolo 12-sexies [...] dopo la parola: 416-bis, sono inserite le seguenti: 452-quater, 452-octies, primo comma». Contrariamente, encontramos las referencias implícitas cuando no existen tales citaciones y a su vez pueden ser tanto internas como externas, en función de si los artículos mencionados forman parte o no de la misma ley de modificaciones. En el primer caso encontramos el art. 452-quinquies, en donde se reflejan otros artículos mencionados líneas antes, «Se taluno dei fatti di cui agli articoli 452-bis e 452-quater è commesso per colpa, le pene previste dai medesimi articoli sono diminuite da un terzo a due terzi»; en el segundo caso, en cambio, encontramos el art. 452-quater, que reza «Fuori dai casi previsti dall'articolo 434, chiunque abusivamente cagiona un disastro ambientale [...]», en donde observamos la presencia del art. 434 que aparece recogido en el Código Penal.

Por último, un caso digno de atención que nos pone en alerta ante la complejidad del proceso de documentación en traducción es el de la multitextualidad, en donde se recogen varios documentos tanto internos como externos, lo cual dificulta la tarea no solo del traductor sino también del jurista. Este es el caso del art. 452-decies, «Le pene previste per i delitti [...] di cui all'articolo 416 aggravato ai sensi dell'articolo 452-octies, nonché per il delitto di cui all'articolo 260 del decreto legislativo 3 aprile 2006, n. 152, e successive modificazioni, [...]». Realizando una búsqueda atenta de las referencias, observamos que el art. 416 aparece en el código penal italiano, el art. 452-octies en la presente ley de modificaciones, y con el DL 152/2006 se establece además una relación de hipertextualidad, ya que es el decreto que dio lugar a la ley (entiéndase la imagen del ordenamiento englobando a la ley como producto final). 
De lo expuesto se desprende que el elemento interdisciplinar es importante en el proceso de documentación, pues el rol de experto otorga al traductor no solo la seguridad temática para afrontar mejor el ejercicio de la profesión, sino también ayuda a encuadrar el proceso de documentación dentro de una realidad concreta. Si bien no es baladí el esfuerzo por parte de las universidades de optar cada vez más por la interdisciplinariedad, es un elemento todavía carente, pues esta supone una gran inversión por la falta de desconfianza entre departamentos. Además, en países como EE. UU. los estudios de derecho se delinean en un postgrado, confiriendo a los estudiantes ya conocimientos previos en otras disciplinas (Doménech 2017). Realizando un breve repaso académico por los dobles Grados que incluyen el derecho como rama interdisciplinar, encontramos entre los más variopintos el Doble Grado en Derecho y Economía, el Doble Grado en Derecho y Filosofía, el Doble Grado en Administración y Dirección de Empresas y en Derecho, el Doble Grado en Derecho y Criminología, el Doble Grado en Humanidades y Estudios Culturales y Derecho, el Doble Grado en Derecho y Periodismo y el Doble Grado en Traducción e Interpretación y en Derecho. En cambio, los planes de estudio que recogen el medio ambiente específicamente y que hemos evidenciado en trabajos anteriores son:

Apréciense, entre muchas otras opciones en España, los Grados universitarios en Ciencias Ambientales, Ciencias del Mar, Ingeniería Forestal, Biología o Geología, entre otros; materias interdisciplinares como Derecho Urbanístico y Ambiental en el Grado en Derecho por la Universidad de Granada; Experto en Gestión Medioambiental en la empresa por la Universidad de la Rioja; Máster en Toxicología Ambiental y Evaluación de Riesgos por la Universidad Juan Carlos I o el Doctorado en Ecología, Conservación y Restauración de Ecosistemas por la Universidad de Alcalá.

Para el caso italiano, en cambio, resaltamos por su interdisciplinariedad los estudios universitarios en Scienze agro-zootecniche de la Universidad de Sassari; en Scienze Naturali de la Universidad de Perugia; en Scienze Ambientali de la Universidad Ca' Foscari de Venecia; en Scienze umane dell'ambiente, del territorio e del paesaggio de la Universidad de Milán; en Ecotossicologia e sostenibilità ambientale de la Universidad de Siena o el Doctorado de Investigación en Scienze della Terra, della Vita e dell'Ambiente de la Universidad de Bolonia. (González Vallejo 2020: 3)

\section{La traducción del medio ambiente}

Las consecuencias evidentes del cambio climático junto a la presión y lucha de las organizaciones no gubernamentales sobre medio ambiente han propiciado la libre circulación de información. A tal respecto, Carrillo y Juan Meinardi (2013) ponen de manifiesto la presencia del llamado "periodismo ambiental" y la necesidad de una traducción específica en este sector que sepa vehicular correctamente los conocimientos aportados. Esto cobra especial relevancia en un panorama internacional en el que la globalización incesante supone una mella en el ecosistema en favor del enriquecimiento económico de los países. A tal respecto, es importante la transparencia y el freno que Organización de Naciones Unidas pone para delimitar las acciones perjudiciales (recuérdese la Directiva 2003/87/CE por la cual se establece el 
comercio de los derechos de emisión entre los países miembros o la instauración del PNUMA para instar a los Gobiernos a la acción contra el cambio climático).

La labor de los lexicógrafos en la redacción del material terminológico sobre medio ambiente se ve obstaculizada por la multidisciplinariedad del sector y la novedad que supone, pues si bien goza de gran popularidad al estar en el candelero político por las cuestiones candentes que provoca, por ejemplo, la contaminación, sus límites son inespecíficos, llegando a representar una "transdisciplina" (Marimón Llorca 2008: 93). Esta autora, en su estudio, pone de relevancia la falta de sistematización de términos en la disciplina, pues los diccionarios de ecología analizados divergen en los términos presentados, lo cual conlleva que presenten un producto con un cariz más divulgativo, a causa de sus múltiples y posibles destinatarios, que específico. Adicionalmente, la terminología que recogen estos instrumentos no está compuesta solamente de palabras en singular, como recoge la norma, sino también de palabras plurales, nombres comunes especificados como desarrollo sostenible, nombres propios como Agenda 21, siglas como CR (Cromo) o EMAS (Eco Managment and Audit Sscheme) y sintagmas terminológicos como bombilla bajo-consumo o economía medioambiental (Marimón Llorca 2008)².

A esto hay que añadir que el imperante dominio del inglés ha proporcionado neologismos que dificultan el trabajo del traductor al carecer de un equivalente exacto en la lengua meta. Algunos de estos ejemplos que han incorporado un correspondiente con carácter de urgencia son eco-friendly (respetuoso con el medio ambiente), littering (desechos abandonados), green (ecológico) y greenwashing (lavado de imagen verde). Por otra parte, el lenguaje del medio ambiente, cual sector especializado, esconde varios niveles terminológicos recurrentes en los textos. Esto nos lleva a hablar de los dos tipos de términos que, según Serianni (2003), encontramos en los textos especializados. Inicialmente, los tecnicismos específicos (tecnicismi specifici) se corresponderían con el sector del medio ambiente; son unívocos, carentes de ambigüedad y desempeñan un fin concreto como approccio ecosistemico, bioaccumulo, carico corporeo, combustibili fossili, diossine, effetto serra, fluorocarburi, glifosato, etc. Por otra parte, nos encontramos con los tecnicismos colaterales (tecnicismi collaterali), los cuales aparecen en un estilo grandilocuente y ampuloso del lenguaje jurídico a través de construcciones como cagionare una compromissione, infliggere una pena o predisporre ostacoli.

Por todo ello, la traducción del medio ambiente se postula como un gran reto para el traductor debido a la novedad de la disciplina, la indefinición terminológica que presenta y la confluencia de sectores presentes que posibilitan su delimitación como ciencia (Arias 2019; Baudo / Lorenzo 2013). A tal propósito, y para que los aspirantes a traductores adquieran la formación específica en el campo medioambiental, Arias (2019) insiste en la consolidación en clase de la llamada "ecoalfabetización" con el fin de facilitar el proceso de documentación, término que indica que una persona adquiere conciencia del medio que le rodea y emprende acciones a nivel personal en aras de su conservación. A tal respecto, la traducción de textos turísticos muestra los elementos naturales del entorno colindante, dado que son considerados la tarjeta de presentación del lugar de interés, función de la que el traductor deberá tener plena conciencia (Durán / Perazzo 2014).

Los ejemplos son nuestros. 
Si exploramos brevemente cómo se ha recogido el medio ambiente en la Carta Magna y en el Código penal italiano y español como respuesta a uno de los pocos problemas que el ser humano no puede controlar hic et nunc, encontramos que ambos países empiezan a mostrar su preocupación por el medio ambiente a partir de la cumbre de Estocolmo en los años 70, en donde se planteó un acuciado interés por el cuidado y tutela del planeta. La Constitución española de 1978 menciona el medio ambiente en el art. 45 con vistas a su incorporación como Estado miembro a la Unión Europea y a la par de todas las conferencias y cumbres internacionales. La Constitución italiana de 1948, en cambio, ya en sus artículos 2, 9 y 32 mencionaba tímidamente el medio ambiente; sin embargo, a partir de los años 80 verá acrecentada su preocupación después de la explosión del reactor 4 de la central de Chernobyl y del caso nacional Eternit (Siracusa, 2015).

$\mathrm{Si}$ bien en ninguno de los dos textos se encuentra una definición precisa de medio ambiente debido al carácter multidisciplinar de la materia (Grassi 2017), a nivel nacional la normativa 2008/99 ya obligada a los estados miembros a la recogida de los daños contra el medio ambiente. España no unificó los perjuicios medioambientales si bien ya la Ley de Protección Atmosférica de 1972 en su art. 3.3 lo ponía de manifiesto, lo cual abre actualmente las puertas a una elevada intertextualidad de leyes heterogéneas entre ellas. Por su parte, Italia los recogió bajo un código en el año 2006, cuando creó el llamado TUA o Codice dell'ambiente a través del DL 152/2006. Este código consta de 318 artículos y recoge la normativa medioambiental, la gestión de residuos, el saneamiento y la protección del suelo y de las aguas, entre otros. Años más tarde, en 2015 saldría la L. 22 de mayo de 2015 núm. 68 que representó una reforma del Codice dell'ambiente y que fue integrada parcialmente en el código penal italiano en el capítulo vi-bis. En el caso español, el medio ambiente aparece recogido en los títulos XVI (capítulos I-V), XVII (capítulos I-II) y en el XXIV (art. 610) (González Vallejo, 2020).

\section{Recursos digitales para la traducción jurídica}

En este apartado práctico hemos recopilado diversos recursos documentales que sirven de referencia para la búsqueda y el estudio terminológico en el campo medioambiental. Inicialmente, se presentan los documentos normativos inherentes a la legislación en materia medioambiental tanto en italiano como en español junto a la base de datos jurídicas. Posteriormente, se recogen las principales revistas jurídicas y divulgativas sobre medio ambiente para conocer los diferentes campos temáticos que se pueden entrelazar en el texto. Asimismo, la aparición de instituciones y páginas web medioambientales aportan una riqueza documental interesante, pues recogen información de principal interés sobre la situación medioambiental. Por último, se ha dedicado un espacio a los recursos terminológicos, como los glosarios monolingües y multilingües, y a las bases terminológicas, que recogen en su seno numerosos términos sectoriales en diversos campos para una mayor confrontación.

\subsection{Bases de datos jurídicas}

Las bases de datos legislativas en el campo medioambiental se encuentran en continua expansión y se presentan como una fuente de acceso documental encuadrada en 
el sistema normativo de referencia al alcance del traductor a través de la consulta de leyes, disposiciones, actos, tratados o acuerdos internacionales, entre otros.

1. ECOLEX (https://www.ecolex.org/es/). Es un base de datos sobre medio ambiente dirigida por la FAO (Organización de Naciones Unidas para la Alimentación y la Agricultura), la UICN (Unión Internacional para la Conservación de la Naturaleza) y el PNUMA (Programa de las Naciones Unidas para el Medio Ambiente). Entre sus objetivos se puede encontrar la perfección de una base de datos jurídica de fácil acceso junto a la creación de una red de asociados y el incremento del conocimiento por parte de la población en materia de legislación medioambiental.

2. DAUE (http://www.ittig.cnr.it/BancheDatiGuide/daue/Index.htm). Compuesto por casi 200 documentos, recoge la recepción en Italia de las leyes medioambientales europeas en materia de aire, agua y de sustancias peligrosas hasta 1997.

3. DoGi (http://www.ittig.cnr.it/dogi/). Representa una base de datos jurídica muy útil para la búsqueda de referencias bibliográficas publicadas en revistas jurídicas italianas. A través de la clasificación que ofrece por materias jurídicas, podemos encontrar que en derecho agrario se han depositado 987 publicaciones.

4. InfoLEGES (http://www.infoleges.it). Es un metamotor legislativo que engloba otros buscadores dando cuenta de casi un millón y medio de documentos. Recoge el anterior DoGi, la normativa comunitaria y la nacional, además de los contratos públicos. Realizando una búsqueda por "inquinamento atmosferico", encontramos 1831 documentos.

5. EUR-Lex (https://eur-lex.europa.eu/homepage.html). Disponible en 24 lenguas oficiales, es una base de datos compuesta por miles de documentos entre los que podemos encontrar tratados, actos jurídicos y acuerdos internacionales. En su buscador el término "medioambiente" encuentra respuesta en 35.303 documentos. Además, ofrece enlaces directos a numerosas instituciones y organismos de la Unión Europea.

6. CENDOJ (http://www.poderjudicial.es/search/indexAN.jsp). Es el Centro de Documentación Judicial y corresponde al órgano técnico del Consejo General del Poder Judicial y recoge las sentencias del Tribunal Supremo, la Audiencia Nacional, el Tribunal Superior de Justicia, la Audiencia Provincial y los Tribunales Militares y Unipersonales.

7. BOE (https://www.boe.es/buscar/legislacion.php). Desde el 2009 se pueden consultar en línea las leyes, las disposiciones y los actos que se publican a nivel autonómico en el territorio español desde 1960 y en Europa desde 1952. Los documentos se encuentran en varios formatos y el buscador permite filtrar a través de la búsqueda avanzada los documentos por rango, fecha de publicación y materia, entre otros. Con solo el término "medio ambiente" aparecen 1065 resultados.

8. Normattiva (https://www.normattiva.it). Con la colaboración de la Cámara de los diputados, el Senado y el Consejo de ministros, es la base de datos legislativa del Diario oficial del estado italiano, la Gazzetta Ufficiale. Recoge más de 200.000 documentos desde el nacimiento del Estado unitario, incluida la época monárquica de 1861 a 1956 y la época republicana desde 1946 hasta 
la actualidad. Como modo de ejemplo, el término "ambiente" nos ofrece un resultado de 2602 documentos.

\subsection{Documentos normativos}

Las normas aparecen reflejadas por competencias específicas dentro de un ordenamiento, como en el caso de los delitos penales divididos a través de Libros, Títulos, Capítulos y Secciones en el CP. En el caso medioambiental, tal y como se ha mencionado anteriormente, en la legislación italiana las diferentes leyes se han unificado, mientras que en la española nos encontramos con un gran conjunto heterogéneo. A continuación, exponemos los reglamentos normativos que más importancia han generado en ambos países:

1. TUA (http://pmbitonto.altervista.org/Testo\%20Unico\%20Ambiente.pdf). Sus siglas corresponden al Testo Unico Ambiente y es el código unificado de leyes medioambientales italiano. Bajo 318 artículos se recoge la evaluación medioambiental, el saneamiento de lugares contaminados, la reducción de la contaminación y la gestión de residuos, entre otros.

2. Ley de 22 de mayo de 2015 núm. 68 (https://www.gazzettaufficiale.it/eli/ id/2015/05/28/15G00082/sg). Supuso una modificación del TUA y fue recogido parcialmente en el código penal italiano.

3. Código penal italiano (http://www.procuragenerale.trento.it/attachments/ article/31/cp.pdf). En su título VI-bis se recoge parcialmente el documento anterior y se menciona el medio ambiente como bien jurídico, la previsión de situaciones perjudiciales contra el medio ambiente y un cambio en el sistema de castigo.

4. Código penal (https://www.boe.es/buscar/act.php?id=BOE-A-1995-25444). En España se carece de un código unificado y lo mencionado penalmente para el medio ambiente aparece recogido en los títulos XVI, XVII y XIV (art. 630). Por ello, citamos las siguientes leyes, que se postulan entre las más importantes a nivel medioambiental emanadas por el Estado.

5. Ley $21 / 2013$ de 9 de diciembre, de evaluación ambiental (https://www.boe. es/buscar/act.php?id=BOE-A-2013-12913). Tiene como finalidad la protección ambiental en el territorio nacional mediante el establecimiento de medidas, planes, proyectos que tengan un impacto significativo.

6. Ley 42/2007 del Patrimonio Natural y de la Biodiversidad (https://www.boe. es/buscar/pdf/2007/BOE-A-2007-21490-consolidado.pdf). Impone el régimen jurídico de la conservación y tutela del patrimonio y de la biodiversidad en el territorio nacional, acogiendo las normas supranacionales de los últimos años.

7. Ley 30/2014 de Parques Nacionales (https://www.boe.es/diario_boe/txt. php?id=BOE-A-2014-12588). Esta ley contempla el binomio parques-Red como una realidad única cercana a la sociedad, concibe el territorio como un ejemplo más de conservación y apoya los principios de colaboración, coordinación y cooperación.

8. Ley 26/2007 de Responsabilidad Medioambiental (https://www.boe.es/buscar/act.php?id=BOE-A-2007-18475). Expone por primera vez un firme es- 
quema de responsabilidad en el derecho ambiental hacia los operadores causantes de los daños y perjuicios contra el medio ambiente

9. Ley 41/2010 de Protección del Medio Marino (https://www.boe.es/buscar/ act.php?id=BOE-A-2010-20050). Representa la adaptación en el ordenamiento español de la Ley marco 2008/56/CE. Su objetivo es crear para 2020 un mantenimiento eficaz del medio marino junto con estrategias para mantener la biodiversidad.

10. Calidad del aire. Ley 34/2007 (https://www.boe.es/buscar/pdf/2007/ BOE-A-2007-19744-consolidado.pdf). Resalta la prioridad de la tutela del aire y de la protección atmosférica tanto a nivel nacional como internacional, debido a las crecientes preocupaciones creadas por los grandes procesos de industrialización.

11. Ley 1/2005 sobre emisión de gases de efecto invernadero (https://www.boe. es/buscar/act.php?id=BOE-A-2005-3941). Supuso una medida para controlar la emisión de gases contaminantes. A través de esta ley, los Estados pueden vender o comprar derechos de emisión excedentes a otros Estados con el objetivo de respetar el Protocolo de Kioto.

\subsection{Material divulgativo temático de principal interés}

Sin menoscabar el material impreso disponible, Internet proporciona una amplia gama de recursos documentales de libre acceso para la adquisición de conocimientos sobre el medio ambiente. En el caso de las revistas, se encuentran tanto de naturaleza jurídica, producto de reflexiones sobre la legislación y la Jurisdicción imperante, como divulgativa, en donde se intercambian ideas o se exponen hechos recientes y de gran interés para el neófito y el docto.

\subsubsection{Revistas jurídicas sobre medio ambiente}

1. Actualidad Jurídica Ambiental (https://www.actualidadjuridicaambiental. com). Creada en 2008, es una revista con periodicidad continuada. A finales del año pasado sumaba ya 4600 publicaciones y en ella se pueden encontrar artículos, comentarios, notas de legislación y notas de jurisprudencia.

2. Revista Iberoamericana de Derecho Ambiental y Recursos Naturales (http:// www.bibliotecadigital.gob.ar/items/show/1810). Con 35 ediciones y de carácter trimestral, la revista difunde el derecho ambiental a través de las informaciones recogidas en Latinoamérica y Europa para afrontar los problemas del medio ambiente y del derecho sostenible.

3. Justicia Ambiental (http://www.revistajusticiaambiental.cl). Creada en 2010 por la ONG FIMA y de periodicidad anual, cuenta con 11 ediciones que buscan fomentar la discusión crítica y los vacíos legales en materia medioambiental.

4. Revista de Derecho Ambiental (https://revistaderechoambiental.uchile.cl). Nacida en el seno de la Facultad de Derecho de la Universidad de Chile, es de carácter semestral y cuenta con 12 números. En ella se presentan doctrinas, jurisprudencias y recensiones.

5. Lexambiente (http://lexambiente.it). Nace en 1998 en manos del magistrado Luca Ramacci. Es una revista trimestral de derecho penal medioambiental 
y cuenta con casi 15.000 documentos en el archivo divididos por materia y categorías.

6. Rivista Giuridica dell'Ambiente (http://rgaonline.it). Nace en 1986 poco después de la constitución del Ministerio de Medio Ambiente y actualmente se publica tanto en formato electrónico como impreso, en donde se recogen comentarios de sentencias, artículos, contribuciones y experiencias administrativas, entre otros.

7. Rivista quadrimestrale di diritto dell'ambiente (http://www.rqda.eu). Creada en 2010 y con 21 ediciones publicadas, se centra no solo en el ámbito nacional, sino que dedica un espacio al medio ambiente internacional junto a novedades ajenas al derecho administrativo.

\subsubsection{Revistas divulgativas sobre medio ambiente}

1. Boletín ciencias de la tierra (https://revistas.unal.edu.co/index.php/rbct). Perteneciente a la Universidad Nacional de Colombia, este año ha publicado el número 47 bajo una gran especificidad de temas como geodinámica, petrografía, mineralogía, vulcanología y estratigrafía ambiental.

2. Retema (https://www.retema.es). De carácter bimestral, nace en 1987 dedicada al sector medioambiental y trata temas como la contaminación y el tratamiento y la gestión de residuos y de las aguas, entre otros.

3. Tecnología ambiental (https://tecnologiaambiental.mx). Motivados por la Cumbre de la Tierra de 1992 celebrada en Río de Janeiro, nace en 1996 para difundir información sobre desarrollo sostenible y el binomio medio ambiente-salud. Actualmente su periodicidad es bimestral.

4. Teorema ambiental (http://www.teorema.com.mx). Se encuentra en línea desde el 2005 y aborda los temas más actuales sobre medio ambiente, en donde podemos destacar la calidad, el agua, la tecnología, las políticas medioambientales y la flora y la fauna.

5. Observatorio medioambiental (https://revistas.ucm.es/index.php/OBMD). Es una revista de periodicidad anual perteneciente a la Universidad Complutense de Madrid. De carácter abierto, ha publicado 22 ediciones que abordan las ciencias relacionadas con el medio ambiente, su interpretación y el desarrollo sostenible.

6. Energia, Ambiente e Innovazione (https://www.eai.enea.it/archivio). Nace en los años 50 en el seno de la ENEA (Agenzia nazionale per le nuove tecnologie, l'energia e lo sviluppo economico sostenibile) y da a conocer sus contribuciones y los resultados de sus investigaciones; su periodicidad es trimestral y da voz a la investigación e innovación científica.

\subsection{Instituciones $y$ asociaciones de medio ambiente}

En relación con los preceptos de nuestra ley que contemplan la salvaguardia y tutela del medio ambiente, las instituciones y asociaciones medioambientales representan a la sociedad civil en la defensa de los elementos naturales del entorno, incrementando la conciencia ciudadana y llevando a cabo proyectos y campañas para prevenir el prejuicio y daño de los ecosistemas. Una de las principales repercusiones de esta 
acción es incluir en el punto de mira político los temas perjudiciales más apremiantes en aras de crear un beneficio común.

1. Greenpeace (https://es.greenpeace.org/es/). Tiene presencia en 55 países y en su sección "trabajamos en" podemos encontrar las áreas (cambio climático, bosque, océanos, democracia y contropoder, desarme y paz y consumismo) en donde se pueden consultar diversos documentos de principal interés.

2. World Wildlife Fund (WWF) (https://www.wwf.es). Fundada en 1961 se encuentra presente en más de 100 países. En la página web se pueden consultar diferentes publicaciones como Revista en acción o Revista Panda 140.

3. PNUMA (Programa de las Naciones Unidades para el Medio Ambiente). (http://www.unenvironment.org/es). Establece la agenda a nivel mundial y da apoyo a los Gobiernos en la lucha por el medio ambiente. En ella se puede acceder al buscador con más de 15.000 documentos, desde documentos e informes hasta herramientas y plataformas.

4. Red amigos de la tierra internacional (https://www.foei.org/es). Nace en 1971 y actualmente la federación cuenta con 73 grupos. Lucha a favor de la justifica social y ambiental y en su página se pueden consultar los informes anuales desde el 2012 hasta el 2014 y las publicaciones anuales desde el 2001.

5. Grupo clima (https://archive.ipcc.ch/index.htm). Fue creado en 1988 con el fin de proporcionar informes de evaluación acerca del estado, las causas y las repercusiones del cambio climático. Actualmente se encuentra en el momento de redacción del sexto informe; sin embargo, se pueden consultar en su página los cinco ya emanados.

6. Amici della terra (http://www.amicidellaterra.it/index.php). Es una asociación sin ánimo de lucro activa desde 1978 enfocada a la protección del medio ambiente y al desarrollo sostenible. Cuenta con más de 150 publicaciones realizadas entre 1970 y 2010 y a través de su página web se pueden consultar todas las conferencias realizadas.

7. Ambientevita (http://www.ambientevita.it). Con sede en Roma, es una asociación sin ánimo de lucro que lucha a favor de la conservación de la naturaleza y la tutela del territorio. Cuenta con varias publicaciones consultables desde el 2000 hasta el 2006.

\subsection{Portales y páginas web medioambientales}

En esta sección se dispone el acceso a los diferentes ministerios para la consulta de la acción gubernamental de ambos países y a los repositorios de las principales autoridades a favor del medio ambiente como la ONU, quien a través del fomento de las agendas mundiales intenta controlar los avances en el panorama internacional.

1. Ministerio para la Transición Ecológica y el Reto Demográfico (https://www. miteco.gob.es/es/). Creado recientemente en el año 2020, es el Ministerio español dedicado al cuidado y tutela del medio ambiente y en él se pueden consultar las guías de actuación específicas, la legislación y las áreas de actividad medioambientales que cubre. Además, expone diversos proyectos de cooperación como los de educación ambiental y una gran muestra de enlaces de interés de ámbito nacional e internacional. 
2. Ministero dell'ambiente e della tutela del territorio e del mare (https://www. minambiente.it). Su origen data de 1986 y es el Ministerio italiano dedicado al medio ambiente. En él se puede consultar un archivo de biblioteca compuesto por cientos de publicaciones clasificables en función del tema que engloban (agua, aire, energía territorio, naturaleza).

3. ONU Programa para el Medio Ambiente (https://www.unenvironment.org/ es). Se postula como la autoridad más importante a favor del medio ambiente. Establece la agenda mundial y asesora a los gobiernos en sus planes de actuación. Entre sus recursos se puede encontrar un buscador con más de 15.000 documentos de libre acceso.

4. InforMEA (https://www.informea.org/es). Es una base de datos dedicada a los acuerdos multilaterales en materia medioambiental. La información se puede filtrar por regiones y en función de los siguientes temas: biodiversidad, clima, gobernanza medioambiental, la tierra y la agricultura, el agua y los productos químicos y desechos.

5. Banco Mundial BIRF-AIF (https://www.bancomundial.org). Creado en 1944 y con sede en Washington, está compuesto por 189 países miembros y sus objetivos se centran en promover el desarrollo sostenible y reducir la pobreza. En su Repositorio de Conocimiento Abierto se encuentran a disposición miles de publicaciones, documentos e informes.

\subsection{Recursos terminológicos}

Si bien en el campo del medio ambiente los recursos de consulta terminológica son mayores en combinaciones más frecuentes, en italiano y español encontramos algunos instrumentos de acceso abierto para otorgar al texto la coherencia terminológica de la que debe disponer. Divididos en dos secciones, se presentan tanto las bases terminológicas internacionales más recurrentes como el IATE o el Eurovoc, entre otros, como glosarios monolingües y multilingües creados por organismos públicos y privados.

\subsubsection{Glosarios monolingües y multilingües}

1. Glossario di termini sull'ambiente (https://www.fimp.pro/images/areetematiche/ambientesalute/Glossario.pdf).

2. Redactado en 2017 por el grupo FIMP, está dirigido a pediatras interesados en conocer los términos más comunes de la contaminación ambiental para conocer sus efectos negativos sobre la salud de los niños.

3. Glosario per l'ambiente e il paesaggio (https://www.isprambiente.gov.it/ files/pubblicazioni/manuali-lineeguida/mlg-78.1-2012-glossario-dinamico. pdf). Fue elaborado en 2012 por el Istituto Superiore per la Protezione e la Ricerca Ambientale (ISPRA) y en él aparecen tanto términos disciplinares como interdisciplinares y pertenecientes a la lengua común.

4. Agenzia Regionale per la Prevenzione e Protezione Ambientale del Veneto (https://www.arpa.veneto.it/servizi-online/glossari-ambientali/glossario-ambientale). Contiene más de 2.200 términos consultables por letras y además cuenta con un glosario de riesgos medioambientales. 
5. Glosario Ambientale (http://www.arpat.toscana.it/glossario-ambientale). Creado por la Agenzia regionale per la protezione ambientale della Toscana (ARPAT), añade la fuente de origen de los términos propuestos junto a las variantes admitidas.

6. Glosario ambiental de Vitalis (http://www.vitalis.net/recursos/glosario-ambiental/). Este glosario recoge los términos del medio ambiente por parte de esta organización ambientalista sin ánimo de lucro. En él aparece un formulario para la petición de inclusión de términos ausentes.

7. Diccionario de tratamiento de las aguas (https://static.lexicool.com/dictionary/JC7NI98588.pdf). Está redactado por M. A. Ricagno en la combinación italiano-español. Cuenta con 450 entradas y en ocasiones con varias correspondencias en español.

8. Diccionario de términos de gestión de residuos sólidos (https://static.lexicool.com/dictionary/TC1WX114968.pdf). Con 1.000 entradas en los idiomas inglés, francés, alemán, italiano y español se centra en el tema del medio ambiente, la energía y las Ciencias de la Tierra.

9. Glosario sobre el cambio climático (http://publications.gc.ca/collections/ collection_2010/tpsgc-pwgsc/S53-44-2010.pdf). Está redactado por el Gobierno de Canadá en inglés, francés, italiano, portugués, rumano, español y gallego.

10. Glosario sobre la energía eólica (http://publications.gc.ca/collections/collection_2012/tpsgc-pwgsc/S53-46-2012.pdf). Está redactado por el gobierno de Canadá en inglés, francés, italiano, portugués, rumano, español y gallego.

11. Glosario sobre la energía de biocombustible (http://publications.gc.ca/collections/collection_2014/tpsgc-pwgsc/S53-50-2014.pdf). Está redactado por el gobierno de Canadá en inglés, francés, italiano, portugués, rumano, español y gallego.

12. Glosario multilingüe sobre desarrollo sostenible (http://www.oqlf.gouv.qc. $\mathrm{ca} /$ ressources/bibliotheque/dictionnaires/panlatin_ddurable_20150330.pdf). Ha sido creado por la oficina de Quebec de la lengua francesa en 2015 y cuenta con 292 términos.

13. SEMIDE (http://www.semide.net/thematicdirs/glossaries/portal_thesaurus). En el tesauro que propone esta asociación euromediterránea en la que participan todos los estados miembros, se pueden consultar los términos por orden alfabético o a través de las 25 temáticas de clasificación.

\subsubsection{Bases terminológicas}

1. IATE (https://iate.europa.eu/home). Creado en 26 idiomas y con casi 8 millones de términos recogidos, esta base terminológica es de acceso abierto y se actualiza diariamente por las diversas instituciones de Luxemburgo y representa un recurso muy útil para los traductores e intérpretes.

2. Eurovoc (https://op.europa.eu/es/web/eu-vocabularies/th-dataset/-/resource/ dataset/eurovoc). Representa un exhaustivo tesauro multilingüe que abarca numerosas disciplinas y actividades en el marco de la UE. Contiene términos en 23 idiomas de la UE y los contenidos del medio ambiente aparecen recogidos a través de las opciones de política del medio ambiente, medio natural y deterioro del medio ambiente. 
3. Eurotermbank (https://www.eurotermbank.com). Con más de 14 millones de términos, las búsquedas se pueden realizar por combinación de idiomas y por temas.

4. Termbank 2.0 (https://www.uibk.ac.at/translation/). Esta base de datos está dirigida principalmente a traductores e intérpretes a través de 30.725 entradas bilingües y multilingües en más de 25 áreas temáticas diferentes. Las lenguas disponibles son inglés, francés, italiano, español y ruso.

\section{Conclusiones}

En estas líneas hemos puesto de manifiesto la importancia de la documentación en el sector del medio ambiente, disciplina todavía por desarrollarse, y la importancia de los textos paralelos a través de los numerosos recursos lingüísticos y terminológicos, pues otorgan una cierta naturalidad al lenguaje jurídico y hacen que este no se presente como producto de meras traducciones literales. A tal propósito, la competencia traductora en el traductor jurídico pasa no solo por dominar con gran fluidez ambos espectros lingüísticos, sino también deberá reconocer y detectar las principales ramas del derecho, los géneros textuales en el que se encuadran los textos con sus características y las principales instituciones reguladoras que se postulan como uno de los cimientos del ordenamiento. Ante esto, resaltamos la gran indefinición del sector medioambiental que engloba numerosas disciplinas. De hecho, en el caso español, no se han unificado las leyes medioambientales en un código único como hizo Italia en el 2006 con el DL 152/2006, lo cual abre las puertas a una búsqueda incesante e intertextual de leyes heterogéneas. En cuanto a la búsqueda terminológica, el medio ambiente supone un reto de gran envergadura, pues los glosarios y diccionarios recogen términos dispares y carecen de uniformidad terminológica.

Por último, ponemos de manifiesto la falta de corpus bilingües en el medio ambiente en la combinación italiano-español, de los que gozan otras lenguas, lo cual resalta aún más la necesidad de recurrir a textos paralelos como material técnico de documentación más allá del mero uso de diccionarios especializados y de crear instrumentos terminológicos en tal dirección.

\section{Referencias bibliográficas}

Arias, Gala (2019): «La ecoalfabetización como base para la enseñanza de la traducción medioambiental», en C. Carrasco, M. Cantarero, C. Díez (eds.), Traducción y sostenibilidad cultural: sustrato, fundamentos y aplicaciones, Salamanca, Ediciones Universidad de Salamanca, pp. 49-54.

Baudo, Lorena Guadalupe / Lorenzo, Gabriela Fernanda (2013): «Medioambiente: lengua de especialidad en traducción e interpretación», en M. C. Dalmagro, A. Parfeniuk (eds.), III Jornadas internacionales sobre medio ambiente y lenguajes: ecolenguas III, $<\mathrm{https://}$ rdu.unc.edu.ar/bitstream/handle/11086/1780/Baudo\%2C\%20Lorenzo.\%20Medio\%20 ambiente $\% 20$ lengua $\% 20 \mathrm{de} \% 20 \ldots$. pdf? sequence $=10 \&$ isAllowed $=\mathrm{y}>$.

Carrillo, María Eugenia / Juan Meinardi, Marcela Eliana (2013): «Ecoperiodismo y ecotraducción: desafíos del traductor en las campañas de concienciación científica», en M. 
C. Dalmagro, A. Parfeniuk (eds.), III Jornadas internacionales sobre medio ambiente y lenguajes: ecolenguas III, <https://rdu.unc.edu.ar/bitstream/handle/11086/1780/Carrillom\%20Juan\%20Meinardim.\%20Ecoperiodismo.pdf?sequence=19\&isAllowed $=\mathrm{y}>$.

Doménech, Gabriel (2017): «La (falta de) interdisciplinariedad en las facultades de derecho españolas», Transjus, (1), pp. 2-11.<http://diposit.ub.edu/dspace/bitstream/2445/123252/1/ Nota_Domenech_1_2017.pdf $>$.

Durán Muñoz, Isabel y Peruzzo, Katia (2014). «Le aree naturali protette e il turismo natura in italiano e spagnolo: un compito semplice per il traduttore?»,Ecozon@: European Journal of Literature, Culture and Environment, 5 (1), pp. 64-83. <https://ebuah.uah.es/ xmlui/bitstream/handle/10017/20565/aree_Duran_Ecozon\%40_2014_vol5.pdf?sequence $=1 \&$ isAllowed $=\mathrm{y}>$.

García, Pilar Elena (2001): «Competencia y actuación en la traducción documental. Traducción, metrópoli y diáspora», en M. A. Vega, R. Martín-Gaitero (eds.), Traducción, metrópoli y diáspora. Las variantes diatópicas de traducción, Madrid, Universidad Complutense de Madrid, pp. 121-131. <https://dialnet.unirioja.es/servlet/articulo?codigo $=4808733>$.

González Vallejo, Rubén (2020): Lenguaje jurídico comparado: Análisis y traducción de los delitos medioambientales del Código penal italiano. Roma: Aracne.

González Vallejo, Rubén (2021): La intertextualidad en el lenguaje jurídico: reflexión y aplicación en una ley medioambiental italiana. Anuari de Filologia. Estudis de Lingüística.

Grassi, Stefano (2017): «Ambiente e costituzione», Rivista quadrimestrale di diritto dell'ambiente, (3), pp. 4-38. <www.rqda.eu/?dl_id=160>.

Kelly, Dorothy A. (2002): «Un modelo de competencia traductora: bases para el diseño curricular», Puentes, (1), pp. 9-20. <http://wpd.ugr.es/ greti/revista-puentes/pub1/02-Kelly. pdf $>$.

Marimón Llorca, Carmen (2008): «Los diccionarios de ecología y medio ambiente o el léxico entre la ciencia y la conciencia», Verba Hispánica, XVI, pp. 91-106. <https://www. researchgate.net/publication/280610791_Los_diccionarios_de_ecologia_y_medio_ambiente_en_espanol_o_el_lexico_entre_la_ciencia_y_la_conciencia $>$.

Marinkovich, Juana (1998): «El análisis del discurso y la intertextualidad», Boletín de Filología, 37 (2), pp. 729-742. <https://boletinfilologia.uchile.cl/index.php/BDF/article/ view/21478/22776>.

Macedo, Alfonso (2008): «La intertextualidad. Cruce de disciplinas humanísticas», Xihmai, 3 (5), pp. 1-9. <https://dialnet.unirioja.es/servlet/articulo?codigo=4953777>.

Mayoral, Roberto (2005): «El polifacetismo del traductor (jurídico y jurado)», en A. C. García de Toro, I. G. Izquierdo (eds.), Experiencias de traducción: reflexiones desde la práctica traductora, Castellón de la Plana, Publicaciones de la Universidad Jaume I, pp. 165-180.

Mendoza, Antonio (1993): «El concepto de intertextualidad en los textos literarios y en las artes visuales: una reflexión didáctica sobre la contigüidad cultural», en A. Rodríguez López-Vázquez (ed.). Didáctica de lenguas y culturas. III Simposio Internacional de la Sociedad Española de Didáctica de la Lengua y la Literatura, Universidad de La Coruña, Servicio de publicaciones, pp. 333-342. < https://ruc.udc.es/dspace/handle/2183/9236>.

Serianni, Luca (2003): Italiani scritti, Bologna, Il Mulino.

Siracusa, Licia (2015): «La legge 22 maggio 2015, n. 68 sugli ecodelitti: una svolta "quasi” epocale per il diritto penale dell'ambiente», Diritto Penale Contemporaneo, (2), pp. 197224. <http://www.ristretti.it/commenti/2015/luglio/pdf3/articolo_siracusa.pdf>. 


\section{Normativa}

Decreto Legislativo n.152/2006. Gazzetta Ufficiale n.88 del 14 de abril de 2006

Ley de 22 de mayo de 2015 núm. 68. Gazzetta Ufficiale n. 122 del 28 de mayo de 2015

Biblioteca de textos legales (2012): Constitución Española, Madrid, Tecnos.

Senato della Repubblica (2012): Costituzione della Repubblica Italiana. $<$ https://www.senato.it/documenti/repository/istituzione/costituzione.pdf $>$. 